\title{
Strong cosmic censorship: The nonlinear story
}

\author{
Raimon Luna, ${ }^{1}$ Miguel Zilhão, ${ }^{2}$ Vitor Cardoso, ${ }^{2,3}$ João L. Costa, ${ }^{4,5}$ and José Natário ${ }^{5}$ \\ ${ }^{1}$ Departament de Física Quàntica i Astrofísica, Institut de Ciències del Cosmos, Universitat de Barcelona, \\ Martí i Franquès 1, E-08028 Barcelona, Spain \\ ${ }^{2}$ CENTRA, Departamento de Física, Instituto Superior Técnico, Universidade de Lisboa, \\ Avenida Rovisco Pais 1, 1049-001 Lisboa, Portugal \\ ${ }^{3}$ Theoretical Physics Department, CERN, CH-1211 Geneva 23, Switzerland \\ ${ }^{4}$ Departamento de Matemática, ISCTE-Instituto Universitário de Lisboa, Avenida das Forças Armadas, \\ 1649-026 Lisboa, Portugal \\ ${ }^{5}$ Center for Mathematical Analysis, Geometry and Dynamical Systems, Instituto Superior Técnico, \\ Universidade de Lisboa, Avenida Rovisco Pais 1, 1049-001 Lisboa, Portugal
}

(Received 30 September 2018; published 15 March 2019)

\begin{abstract}
A satisfactory formulation of the laws of physics entails that the future evolution of a physical system should be determined from appropriate initial conditions. The existence of Cauchy horizons in solutions of the Einstein field equations is therefore problematic and expected to be an unstable artifact of general relativity. This is asserted by the strong cosmic censorship conjecture, which was recently put into question by an analysis of the linearized equations in the exterior of charged black holes in an expanding universe. Here, we numerically evolve the nonlinear Einstein-Maxwell-scalar field equations with a positive cosmological constant, under spherical symmetry, and provide strong evidence that mass inflation indeed does not occur in the near extremal regime. This shows that nonlinear effects might not suffice to save the strong cosmic censorship conjecture.
\end{abstract}

DOI: $10.1103 /$ PhysRevD.99.064014

\section{INTRODUCTION}

The strong cosmic censorship (SCC) conjecture embodies the expectation that general relativity is a deterministic theory. It does so by predicting that Cauchy horizons $(\mathrm{CH})$ - the boundaries of the maximal evolution of initial data via the Einstein field equations-are unstable and give rise, upon perturbation, to singular boundaries, beyond which the field equations cease to make sense.

Quite surprisingly, recent results [1-8] put into question the validity of SCC in the context of highly charged black holes (BHs) immersed in a spacetime with a positive cosmological constant. ${ }^{1}$ More precisely, these results provide evidence for the existence of stable (charged de Sitter) $\mathrm{BH}$ configurations containing a $\mathrm{CH}$ in their interior, along which the Misner-Sharp mass, a scalar invariant

\footnotetext{
${ }^{1}$ These results appeared almost two decades after the pioneering work in $[9,10]$, which made contradicting claims; see Sec. 2.2 in [6] for a clarification of the implications of these papers regarding SCC.

Published by the American Physical Society under the terms of the Creative Commons Attribution 4.0 International license. Further distribution of this work must maintain attribution to the author(s) and the published article's title, journal citation, and DOI.
}

measuring the energy content of symmetry spheres, remains bounded-a no-mass-inflation scenario. In particular, the $\mathrm{CH}$ should then retain enough regularity to allow evolving the spacetime metric across it using the Einstein equations. However, such evolution is not unique, condemning determinism (and the SCC conjecture) to failure.

This is particularly disturbing in view of the fact that a positive cosmological constant provides the standard mechanism to model the observed accelerated expansion of our Universe. Nonetheless, the results mentioned above are restricted to either the linear setting or to the nonlinear analysis of the geometry of the BH interior starting from an already completely formed event horizon-i.e., the corresponding (horizon) data are put in "by hand". These results provide clear expectations concerning the stability/instability of Cauchy horizons within de Sitter $\mathrm{BHs}$, but are not enough to cast a final verdict on SCC for the following reasons: first, the parameter ranges identified in [4] as potentially problematic for SCC are very narrow, and therefore even small nonlinear deviation from these might be enough to save SCC; secondly the (nonlinear) results in [1] do not take into account the oscillatory behavior of the scalar field along the event horizon, ${ }^{2}$ which was identified in [4] for the first time. Moreover,

\footnotetext{
${ }^{2}$ In [1] it is only considered the case where the (real) scalar field satisfies $\phi_{, v} \sim e^{-k v}$, along the event horizon.
} 
our numerical simulations will allow us to gain further information concerning the behavior of various relevant quantities near the Cauchy horizon, as for instance a quantitative understanding of tidal deformations.

To go beyond the previous studies we perform a full nonlinear numerical evolution of both massless and massive minimally coupled self-gravitating scalar fields, in a spacetime with a Maxwell field and a positive cosmological constant. For technical reasons we will restrict ourselves to the spherically symmetric setting, but we should stress that, according to the results in [4], the spherically symmetric mode plays a key role in the linear stability/instability of Cauchy horizons for near extremal Reissner-Nordström de Sitter BHs.

All the nonlinear simulations we will be discussing evolve from characteristic initial data whose outgoing component is located in the $\mathrm{BH}$ exterior; in particular, the data along the event horizon arises dynamically in this framework. Our numerical code also allows us to probe the $\mathrm{BH}$ interior region and to derive a detailed description of the behavior of fundamental quantities, such as the radius function, scalar field, (Misner-Sharp) mass, and curvature. By examining the vicinity of the $\mathrm{BH}$ parameters identified as potentially problematic for SCC in [4], we find stable no-mass-inflation scenarios arising from a full nonlinear evolution (of exterior data). These are, to the best of our knowledge, the first results of this kind. They show, in particular, that nonlinear effects are apparently not strong enough to save SCC in the context of highly charged de Sitter BHs. ${ }^{3}$

Our setup also allows the inclusion of a scalar field mass, and so we take the opportunity to investigate the possibility, raised in $[5,6]$, that the curvature might be bounded up to the Cauchy horizon for certain choices of this mass. This scenario is disproved in the cases that we analyze.

\section{SETUP}

We consider here an evolving, electrically charged spacetime, modeled by the Einstein-Maxwell action with a cosmological constant $\Lambda$, minimally coupled to a massive scalar field $\Phi$ with mass parameter $\mu$,

$$
S=\int d^{4} x \sqrt{-g}\left(R-2 \Lambda-F^{2}-2 \Phi_{, \alpha} \Phi^{, \alpha}-2 \mu^{2} \Phi^{2}\right),
$$

where $F^{2}=F_{\alpha \beta} F^{\alpha \beta}$ and $F_{\alpha \beta}$ is the Maxwell tensor. The equations of motion reduce to

$$
\begin{aligned}
G_{\mu \nu}+\Lambda g_{\mu \nu}= & 2 F_{\mu \alpha} F_{\nu}{ }^{\alpha}-\frac{1}{2} g_{\mu \nu} F^{2}+2 \Phi_{, \mu} \Phi_{, \nu} \\
& -g_{\mu \nu}\left(\Phi_{, \alpha} \Phi^{, \alpha}+\mu^{2} \Phi^{2}\right)
\end{aligned}
$$

\footnotetext{
${ }^{3}$ It should be noted that, in view of recent developments [11-16], the situation concerning SCC in the context of asymptotically flat $(\Lambda=0) \mathrm{BHs}$ is far more clear.
}

$$
\square \Phi=\mu^{2} \Phi, \quad d F=d \star F=0,
$$

where $\star$ is the Hodge dual.

We focus on spherically symmetric spacetimes, written in double null coordinates as

$$
\begin{gathered}
d s^{2}=-2 e^{2 \sigma(u, v)} d u d v+r^{2}(u, v) d \Omega^{2}, \\
F=F_{u v}(u, v) d u \wedge d v, \quad \Phi=\Phi(u, v),
\end{gathered}
$$

where $u$ and $v$ are ingoing and outgoing coordinates, respectively. In this framework, Maxwell's equations decouple and imply that

$$
F_{u v} r^{2} e^{-2 \sigma}=\text { constant }=Q,
$$

with $Q$ a conserved (electric) charge.

\section{NUMERICAL EVOLUTIONS}

To numerically evolve the field equations, we specify initial conditions along two null segments, $u=u_{i}$ and $v=v_{i}$. We fix the residual gauge freedom as follows:

$$
r\left(u_{i}, v\right)=v, \quad r\left(u, v_{i}\right)=r_{0}+u r_{u 0},
$$

where $r_{u 0}$ is a constant and $r_{0}=v_{i}$. The profile of the scalar field is set as purely ingoing,

$$
\Phi\left(u_{i}, v\right)=A e^{-\left(\frac{v-v_{c}}{w}\right)^{2}},
$$

with the outgoing flux being set to zero, $\Phi_{, u}\left(u, v_{i}\right)=0$. See Appendix A for more information about the integration procedure.

To interpret our results it will be convenient to consider the following alternative outgoing null coordinates: $\stackrel{\circ}{v}$, an Eddington-Finkelstein type coordinate, defined by integrating

$$
\left(1-\frac{2 M}{r}+\frac{Q^{2}}{r^{2}}-\frac{\Lambda}{3} r^{2}\right) d v=r_{, v} d v
$$

along the event horizon (EH), and $t$, the affine parameter of an outgoing null geodesic, obtained by integrating

$$
\left(1-\frac{2 M}{r}+\frac{Q^{2}}{r^{2}}-\frac{\Lambda}{3} r^{2}\right) d t=-r_{, u} r_{, v} d v
$$

along a constant $u$ line. In these expressions $M$ stands for the Misner-Sharp mass function, which we also closely monitor during the integration, given by 


$$
M(u, v)=\frac{r}{2}\left(1+\frac{Q^{2}}{r^{2}}-\frac{\Lambda}{3} r^{2}+2 e^{-2 \sigma} r_{, u} r_{, v}\right) .
$$

The constant $r_{u 0}$ is thus related to the initial $\mathrm{BH}$ mass, $M_{0} \equiv M\left(u_{i}, v_{i}\right)$. Recall that the blowup of this scalar signals the breakdown of the field equations [17] (compare with $[12,13])$.

To estimate the curvature we compute the Kretschmann scalar $K$ computed from the field equations (a direct evaluation of this scalar in terms of the metric was found to lead to important round-off errorrelated problems).

According to the results in Refs. [1,4], concerning the massless case, we expect the curvature to blow up for all nontrivial initial data throughout the entire subextremal parameter range. Although it is a potentially interesting nonlinear effect, we recall that the blowup of $K$, per se, is of little significance: it implies neither the breakdown of the field equations [18] nor the destruction of macroscopic observers [19]. Recall that the results in Refs. [5,6] suggest that the introduction of scalar mass could lead, for appropriate choices of $\mathrm{BH}$ parameters, to solutions with bounded curvature. As we will see below, our results contradict this expectation.

\section{INITIAL CONDITIONS}

The physical problem is then fully determined upon specifying $Q, \Lambda, \mu, M_{0}, A, v_{c}$, and $w$. Since our purpose here is to determine whether the linearized predictions of Refs. $[4,5]$ hold in the full nonlinear regime, we focus on $M_{0}=1, \Lambda=0.06$ and use the following configurations:

(A) $Q=0.9000, \mu=0$, corresponding to $Q=0.890 Q_{\max }$. In this case, the results in Ref. [4] (lower left panel of Fig. 3) predict mass inflation.

(B) $Q=1.0068, \mu=0$, corresponding to $Q=0.996 Q_{\max }$. Linearized studies provide evidence in favor of a no-mass-inflation scenario [4].

(C) $Q=1.0068, \mu=1.0$. The results of Ref. [4] together with those of Ref. [5] (see Fig. 2) also provide evidence in favor of a no-mass-inflation scenario. Here we are considering the superposition of both neutral massless scalar perturbations [4] and charged massive scalar perturbations [5] as being the most predictive of the full nonlinear evolution. If we just take into account massive scalar perturbations, then the results in Ref. [5] (see Fig. 2) and [6] (p. 22) suggest that curvature might also be bounded.

To test the dependence of our results on initial data, we use the following initial profiles for the scalar field:

(1) $A=0.04, w=0.1$, and $v_{c}=3.0$;

(2) $A=0.08, w=0.5$, and $v_{c}=3.0$.

We have evolved the relevant system of equations using the DoNuTS code. It is based on the formulation presented in Refs. [20-22], but the integration technique makes it

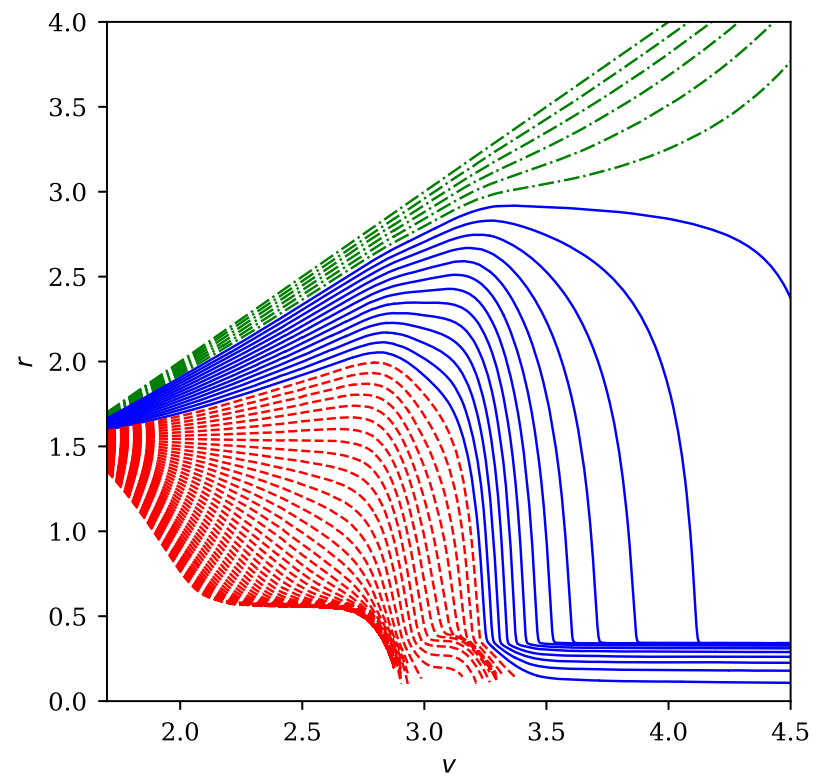

FIG. 1. Radius function for constant- $u$ slices in a configuration with $M_{0}=1.0, Q=0.9, \Lambda=0.06, \mu=0, A=0.4, v_{c}=3.0$, and $w=0.25$. Dashed-dotted green lines reach infinity, full blue lines hit the $\mathrm{CH}$ and red dashed lines hit the singularity at $r=0$.

spectrally accurate in the $v$-direction and, correspondingly, runs with trivial memory requirements and orders of magnitude faster than previously reported codes.

\section{RESULTS}

It is important to start by noticing that, as widely expected [1,11], our numerics show that all solutions contain a nonempty $\mathrm{CH}$ in their $\mathrm{BH}$ interior. This can be attested by monitoring the radius function (shown in Fig. 1) along null lines $u=u_{1}$, for $u_{1}>u_{\mathrm{EH}}$, where $u=u_{\mathrm{EH}}$ is the event horizon. In fact, for $u_{1}$ larger but close to $u_{\mathrm{EH}}$, the radius converges, in $v$, to a nonvanishing constant. It is also interesting to note that, for some initial configurations and large enough $u_{1}$, the radius does converge to zero, signaling (in that region) a singularity beyond which the metric cannot be extended [23].

As is well known, the behavior of the scalar field along the event horizon is of great significance for the structure of the $\mathrm{BH}$ interior region. The first noteworthy feature of our results is that, as expected, the field decays exponentially (in $\stackrel{\circ}{v}$ ). More surprisingly, we also clearly observe an oscillatory profile; this might seem odd at first, since it is in contrast with what happens for $\Lambda=0$ and with the expectation created by the study of sufficiently subextremal BHs with $0<M^{2} \Lambda \ll 1$ [24]. However, it turns out that such behavior should be expected from the linearized analysis of Refs. [4,5], where it is shown that, for a configuration resembling our configuration $\mathbf{B}$, there are two modes which dominate the response: a nonoscillatory "near extremal" (NE) mode with characteristic frequency $\omega_{\mathrm{NE}} \sim-0.081 i$ and a "photon sphere" (PS) mode with 


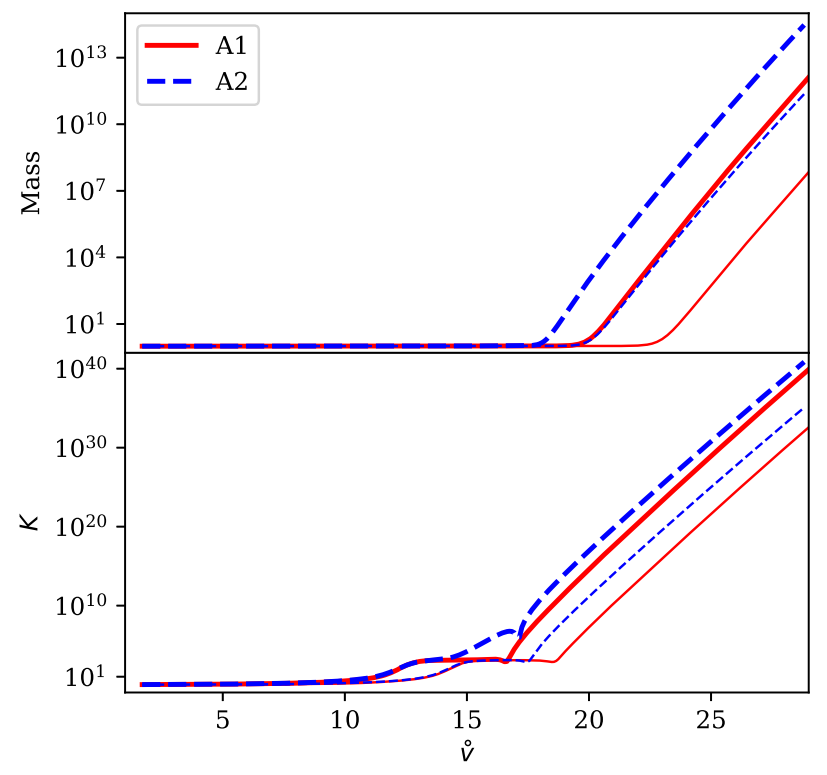

FIG. 2. Mass function (10) and Kretschmann scalar as functions of $\dot{v}$ for configurations A1 (red solid line) and A2 (blue dashed line). Thin lines are evaluated at $u=u_{\mathrm{EH}}+1$ and thick lines are evaluated at $u=u_{\mathrm{EH}}+2$. These results are consistent with the existence of mass inflation leading to a weak singularity.

$\omega_{\mathrm{PS}} \sim 0.096-0.095 i$ (these numbers are given in the units and time coordinate of Ref. [4]). Here we find very good agreement with the PS mode (when translated to our $\stackrel{v}{v}$ coordinate) which is oscillatory in nature. Similar agreement can be found for the remaining configurations $\mathbf{A}$ and C. We also recall that, according to the results in [4], in the $M^{2} \Lambda \ll 1$ case, the dominant mode is a nonoscillatory "de Sitter" mode, in agreement with [24].

Our main results (concerning mass and curvature) are summarized in Figs. 2-4. Figure 2 shows the evolution of the mass function and the Kretschmann scalar for configurations A: in these cases, mass inflation occurs, and, consequently, the curvature invariant $K$ diverges. Note that an observer crossing one such region will be subjected to physical deformations which are not necessarily infinite (see discussion below). Nonetheless, because there is mass inflation, the singularity is strong enough to deserve the classification of "terminal boundary", since it corresponds to a locus where the field equations cease to make sense. These conclusions are consistent with the linear results in [4] and the nonlinear results in [1].

The main novel result of this work concerns Fig. 3: there exist configurations for which no mass inflation occurs. For configurations $\mathbf{B}$, in the $\mathrm{BH}$ interior, after a small "accretion" stage, the mass settles to a constant value. Moreover, as recently predicted $[1,4]$, the $\mathrm{CH}$ remains a curvature singularity, since the curvature scalar $K$ diverges. However, the lack of mass inflation makes the singularity so "mild" that, in principle, one should be able to continue the evolution of the space-time metric across it, by solving the Einstein field equations.

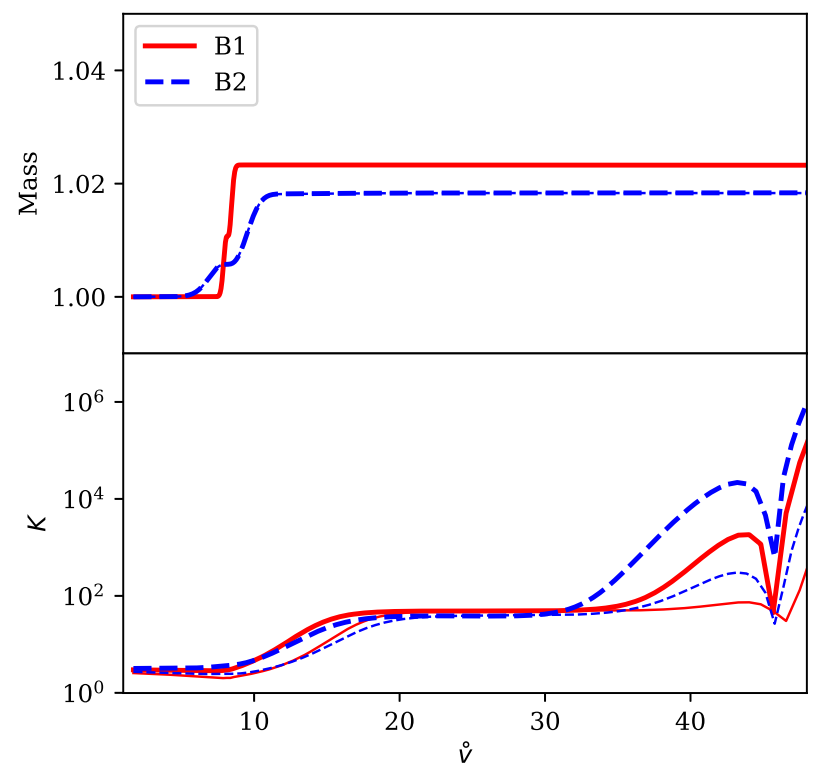

FIG. 3. Mass function (10) and Kretschmann scalar as functions of $\stackrel{\circ}{v}$ for configurations B1 (red solid line) and B2 (blue dashed line). Thin lines are evaluated at $u=u_{\mathrm{EH}}+1$ and thick lines are evaluated at $u=u_{\mathrm{EH}}+2$.

A somewhat unexpected feature (of configuration $\mathbf{B}$ ) is the oscillatory way in which the curvature scalar diverges. In hindsight, such behavior could be expected from the previously discussed oscillatory behavior of the scalar field along the event horizon. Note that in a no-mass-inflation situation it is the blowup of $\Phi_{, v} / r_{, v}$ that dominates the behavior of $K$. This should be contrasted with what happens when mass inflation occurs: then it is the monotone divergence of the mass that controls the Kretschmann; this last fact provides an explanation for the nonoscillatory behavior observed for configuration $\mathbf{A}$.

Concerning massive scalars, the results presented in Fig. 4 identify configuration $\mathbf{C}$ as another no-mass-inflation configuration. Once again we find that the corresponding $\mathrm{CH}$ is a "weak" curvature singularity. In fact, the presence of the scalar mass seems to have no attenuation effect on the growth of $K$, in contrast with what might be expected from the linear analysis in $[5,6]$.

We finish this section with some further remarks concerning the blowup of curvature. In configuration $\mathbf{A}$, our results indicate that the Kretschmann scalar blows up as $t^{-2}$ (possibly modulated by logarithmic terms), where $t$ is the affine parameter defined in (9) with the Cauchy horizon located at $t=0$. This might suggest that the curvature blows up as $t^{-1}$, but, as noted in $[19,25]$, there are curvature components that may blow up even faster. In fact, the quantities that determine the blowup of the Kretschmann scalar are the square of the (Misner-Sharp) mass $M$ and the square of the gradient of $\Phi$, which is dominated by $\left(\Phi_{, v} / r_{, v}\right)^{2}$. However, all curvature components are controlled by $M$ and $\left(\Phi_{, v} / r_{, v}\right)^{2}$ (the origin of the last term can be traced to the energymomentum tensor). From the behavior of the Kretschmann 


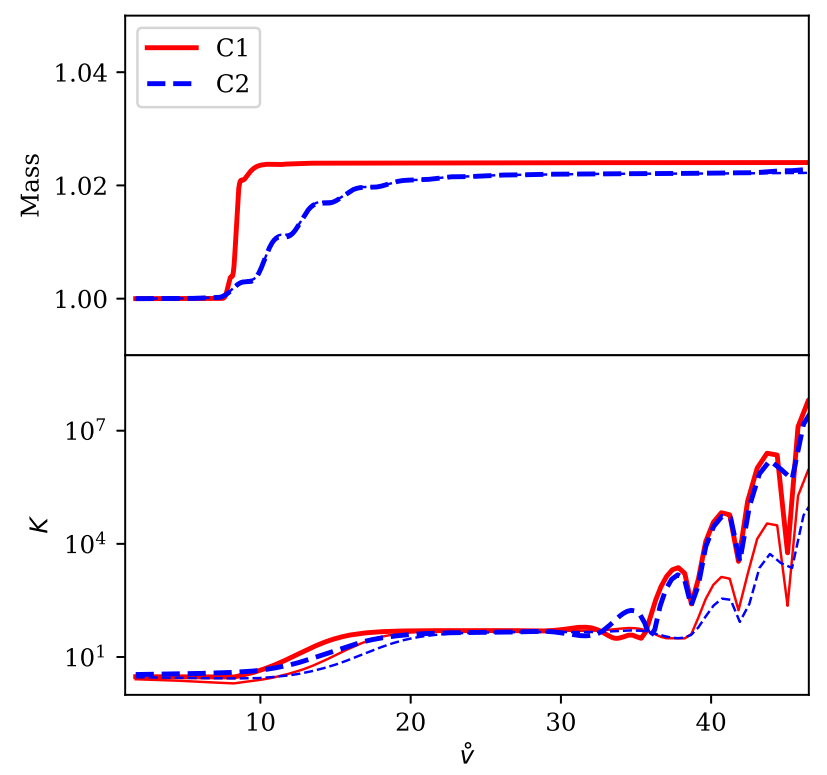

FIG. 4. Same as Fig. 3, for configurations $\mathbf{C 1}$ (red solid) and C2 (blue dashed).

scalar, we can then conclude that the components of the curvature blow up at most as $t^{-2}$; we also expect inverse logarithmic powers $[12,13,19]$ that are hard to detect numerically. Although divergent, these curvature components should yield (with the help of the logarithmic terms) a finite "tidal deformation" when integrated twice with respect to $t$, in agreement with the picture in [19].

From the equation (see Ref. [26])

$$
M_{, v}=\frac{1}{2}\left(1-\frac{2 M}{r}+\frac{Q^{2}}{r^{2}}-\frac{\Lambda}{3} r^{2}\right)\left(\frac{\Phi_{, v}}{r_{, v}}\right)^{2} r_{, v},
$$

we conclude that no mass inflation is essentially equivalent to the integrability of $\left(\Phi_{, v} / r_{v}\right)^{2}$, with respect to $t$. $^{4}$ Moreover, when both occur we immediately see that the curvature can only give rise to finite "tidal deformations." This reasoning is verified by our results concerning configuration $\mathbf{B}$, for which the mass is bounded, $\Phi_{, v} / r_{, v}$ grows slower than $t^{-1 / 2}$, and the Kretschmann scalar and the curvature components blow up at most as $t^{-1}$.

\section{DISCUSSION}

The main motivation for this study was to understand whether nonlinear effects could trigger mass inflation, even when the linearized analysis suggests otherwise [4]. We found that nonlinear effects are not strong enough to change the picture: in fact, the nonlinear results are in full agreement with the linearized predictions. The linearized

\footnotetext{
${ }^{4}$ In fact, there is a mathematical equivalence under the reasonable, in principle generic, assumption that the quantity $1-\frac{2 M}{r}+\frac{Q^{2}}{r^{2}}-\frac{\Lambda}{3} r^{2}$ does not vanish at the Cauchy horizon.
}

analysis of Ref. [4] suggests that no mass inflation should occur for BH charge above a threshold $Q_{*} \simeq 0.95$. Within a nonlinear evolution, the precise linearized results are difficult to reproduce (for instance, the final spacetime parameters depend on the initial parameters and on the size of the initial data). However, for small scalar amplitudes, our results are indeed consistent with the previous threshold.

The (numerical) no-mass-inflation solutions presented here are the first solutions of this kind arising from the full nonlinear evolution of exterior data. They contain a Cauchy horizon in their $\mathrm{BH}$ interior region that can be seen as ("weakly") singular, due to the divergence of curvature invariants. However, these divergent tidal forces are not necessarily strong enough to lead to a divergent tidal deformation and the consequent unequivocal destruction of all macroscopic objects [19]. Even more problematic, the lack of mass inflation indicates that these Cauchy horizons should maintain enough regularity as to allow the field equations to determine (classically), in a highly nonunique way, the evolution of the metric to their future. This corresponds to a potential severe violation of SCC.

Our results concern spherically symmetric spacetimes. The picture is unlikely to change even with asymmetric initial conditions [4]. Thus, from the conceptual point of view [5], our results show that SCC is not enforced by the field equations. In the meantime, interesting suggestions to remedy SCC, in the presence of a positive cosmological constant, have been put forward: these include enlarging the allowed set of initial data by weakening their regularity [27] or restricting the scope of SCC to the uncharged $\mathrm{BH}$ setting [28]. It thus seems plausible that the astrophysical interpretation of SCC remains valid, once other fields and realistic $\mathrm{BH}$ charges are considered.

\section{ACKNOWLEDGMENTS}

We are indebted to Kyriakos Destounis, Aron Jansen, and Peter Hintz for many and very useful conversations and comments. R. L. gratefully acknowledges the hospitality of the group at CENTRA, where this work was started. We are grateful to the Yukawa Institute for Theoretical Physics at Kyoto University, for hospitality while this work was completed during the YITP-T-18-05 on "Dynamics in Strong Gravity Universe." R. L. is supported by Ministerio de Educación, Cultura y Deporte Grant No. FPU15/01414. M.Z. acknowledges financial support provided by FCT/ Portugal through the IF program, Grant No. IF/00729/ 2015. J. L.C. and J.N. acknowledge financial support provided by FCT/Portugal through UID/MAT/04459/ 2013 and Grant No. (GPSEinstein) PTDC/MAT-ANA/ 1275/2014. The authors acknowledge financial support provided under the European Union's H2020 ERC Consolidator Grant "Matter and strong-field gravity: New frontiers in Einstein's theory" Grant No. MaGRaTh646597. This project has received funding from the European Union's Horizon 2020 research and innovation program under the Marie Sklodowska-Curie Grant 
No. 690904. The authors would like to acknowledge networking support by the GWverse COST Action CA16104, "Black holes, gravitational waves and fundamental physics." This research was supported in part by Perimeter Institute for Theoretical Physics. Research at Perimeter Institute is supported by the Government of Canada through the Department of Innovation, Science, and Economic Development, and by the Province of Ontario through the Ministry of Research and Innovation.

\section{APPENDIX A: NUMERICAL PROCEDURE}

\section{Algorithm}

Our equations of motion have the form

$$
\begin{aligned}
r_{, u v}+\frac{r_{, u} r_{, v}}{r}+\frac{e^{2 \sigma}}{2 r}\left[1-\frac{Q^{2}}{r^{2}}-\left(\Lambda+\mu^{2} \Phi^{2}\right) r^{2}\right] & =0, \\
\sigma_{, u v}-\frac{r_{, u} r_{, v}}{r^{2}}-\frac{e^{2 \sigma}}{2 r^{2}}\left(1-2 \frac{Q^{2}}{r^{2}}\right)+\Phi_{, u} \Phi_{, v} & =0, \\
\Phi_{, u v}+\frac{1}{r}\left(\Phi_{, u} r_{, v}+\Phi_{, v} r_{, u}\right)+\frac{e^{2 \sigma}}{2} \mu^{2} \Phi & =0,
\end{aligned}
$$

and are subjected to the following constraints:

$$
\begin{aligned}
& r_{, u и}-2 r_{, u} \sigma_{, u}+r\left(\Phi_{, u}\right)^{2}=0, \\
& r_{, v v}-2 r_{, v} \sigma_{, v}+r\left(\Phi_{, v}\right)^{2}=0 .
\end{aligned}
$$

These equations must be satisfied by the initial data. Then, by virtue of the Bianchi identities, they will be satisfied in the whole computational domain provided that the dynamical equations are accurately satisfied.

To integrate these equations, we start by transforming them into a system of ordinary differential equations (ODEs). Our procedure is as follows. Let $h(u, v)$ be any evolved quantity $r(u, v) ; \sigma(u, v)$; and $\Phi(u, v)$. Defining $f(v)=\partial_{u} h(u, v)$, all dynamical equations, for fixed $u$, have the form

$$
f^{\prime}(v)+f(v) p(v)=g(v)
$$

where / denotes the derivative with respect to $v$. These equations can be solved by introducing the integrating factor

$$
\lambda(v)=\exp \left(\int_{v_{i}}^{v} p\left(v^{\prime}\right) d v^{\prime}\right), \quad \lambda^{\prime}(v)=p(v) \lambda(v) .
$$

Multiplying Eq. (A6) by $\lambda(v)$, we get

$$
\begin{aligned}
f^{\prime}(v) \lambda(v)+f(v) \lambda^{\prime}(v) & =[f(v) \lambda(v)]^{\prime}=g(v) \lambda(v) \\
\Leftrightarrow f(v) \equiv \partial_{u} h(v) & =\frac{1}{\lambda(v)}\left[f\left(v_{i}\right)+\int_{v_{i}}^{v} g\left(v^{\prime}\right) \lambda\left(v^{\prime}\right) d v^{\prime}\right],
\end{aligned}
$$

which are ODEs in $u$ for all values of $v$. Given initial conditions in the two null segments $u=u_{i}, h\left(u_{i}, v\right) \forall v$ and $v=v_{i}, f\left(v_{i}\right) \equiv \partial_{u} h\left(u, v_{i}\right) \forall u$, we can integrate the equations in a rectangular region $u_{i}<u<u_{f}$ and $v_{i}<v<v_{f}$.

For our three functions in Eqs. (A1)-(A3), $p(v)$ and $g(v)$ are the following:

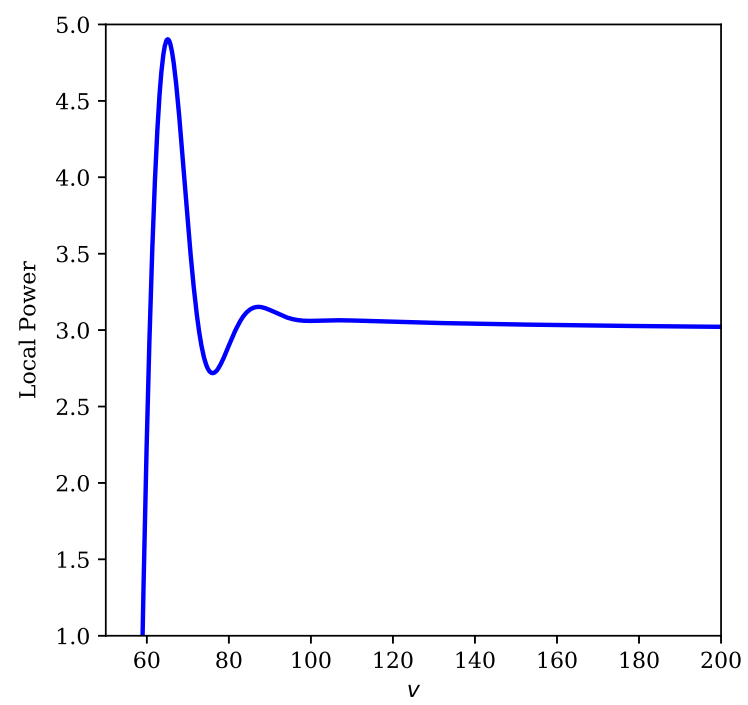

FIG. 5. Massless scalar field along the event horizon with corresponding "local power" for a configuration with $M_{0}=1.0, Q=0.95$, $\Lambda=0, \mu=0, A=0.01, v_{c}=6.0$, and $w=0.25$. The power-law decay $\Phi \sim v^{-3}$ matches to a very good precision the one expected from linearized analysis [32] and reproduces well previous nonlinear results [20]. 


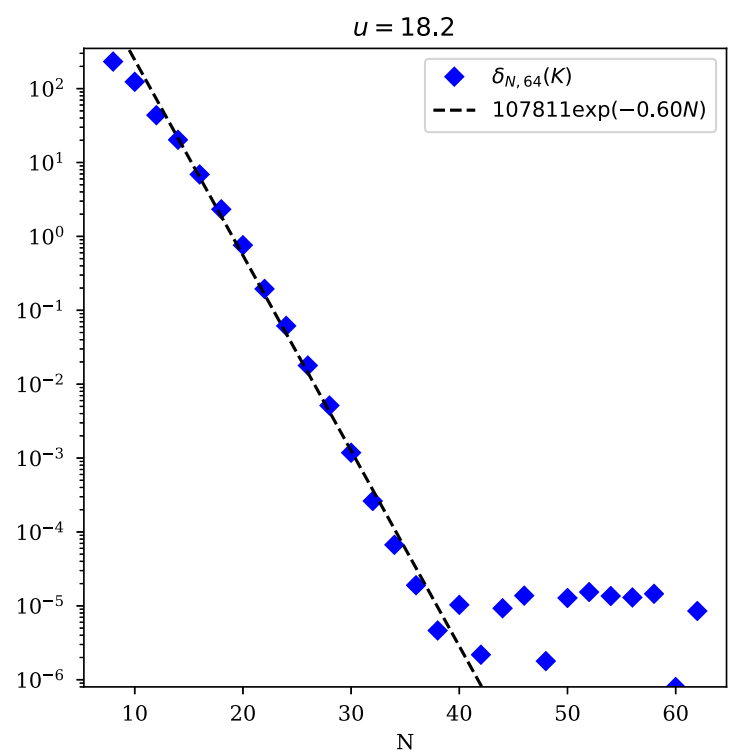

FIG. 6. $\delta_{N, 64}(K)$ at $u=18.2$ for configuration B1. 20 domains were employed in the $v$ direction, where each domain has $N$ points. The plot clearly shows exponential convergence until $N \approx 40$.

$$
\begin{aligned}
& p_{r}(v)=\frac{r_{, v}}{r}, \\
& g_{r}(v)=-\frac{e^{2 \sigma}}{2 r}\left[1-\frac{Q^{2}}{r^{2}}-\left(\Lambda+\mu^{2} \Phi^{2}\right) r^{2}\right], \\
& p_{\Phi}(v)=\frac{r_{, v}}{r} \\
& g_{\Phi}(v)=-\frac{r_{, u} \Phi_{, v}}{r}-\frac{e^{2 \sigma}}{2} \mu^{2} \Phi, \\
& p_{\sigma}(v)=0, \\
& g_{\sigma}(v)=\frac{r_{, u} r_{, v}}{r^{2}}+\frac{e^{2 \sigma}}{2 r^{2}}\left(1-2 \frac{Q^{2}}{r^{2}}\right)-\Phi_{, u} \Phi_{, v} .
\end{aligned}
$$

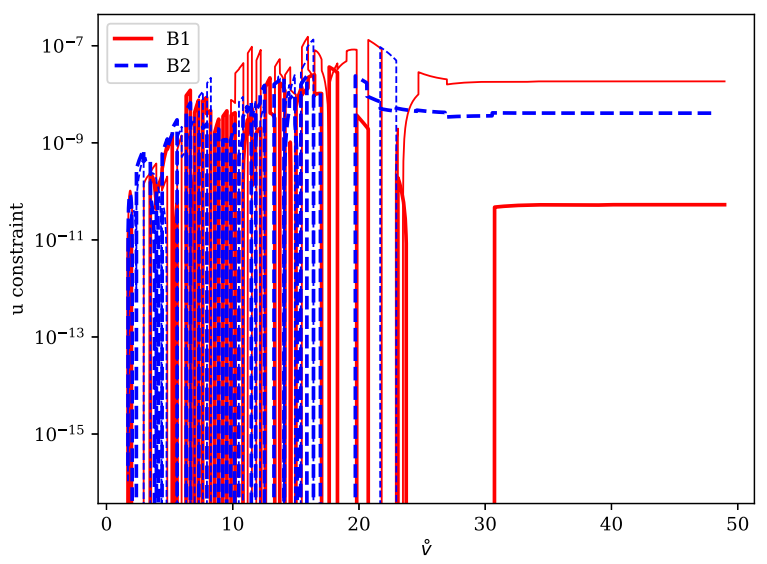

We integrate these equations using the Double Null Through Spectral Methods (DoNuTS) code written in Julia [29]. To integrate the system within DoNuTS, all functions are expanded in a Chebyshev basis in the $v$ direction (where all $v$ derivatives and integrations can be readily performed), and the remaining ODEs in the $u$ direction are integrated using an adaptive step integrator through the DifferentialEquations.jl Julia package [30].

\section{Adaptive gauge}

When using the initial gauge, $r_{u}$ becomes extremely large around the apparent horizon for large $v$. Therefore, in order to explore the near-horizon region at late times, it is convenient to use an adaptive gauge in $u$ during the numerical evolution.

Since the change $u \rightarrow \tilde{u}(u)$ together with $\sigma \rightarrow \sigma-\frac{1}{2} \log \left(\frac{d \tilde{u}}{d u}\right)$ leaves the equations invariant, we can change the gauge in $u$ along the integration by choosing appropriately the initial condition $\sigma_{, u}\left(u, v_{i}\right)$ at each value of $u$.

To explore the near-horizon geometry, we can choose an Eddington-like gauge for $u$, i.e., a gauge that brings the event horizon to $u \rightarrow \infty$. A good way to do so, as described in [31], is to set $\sigma\left(u, v_{f}\right)=\log \left(2 r_{, v}\left(u, v_{f}\right)\right)+C$, where $C$ can be any constant. In the DoNuTS code, this is achieved by picking the initial condition for $\sigma_{, u}\left(u, v_{i}\right)$ :

$$
\sigma_{, u}\left(u, v_{i}\right)=-\left[\sigma\left(u, v_{f}\right)-\log \left(2 r_{, v}\left(u, v_{f}\right)\right)+\frac{3}{2} \log 2\right] .
$$

The term $\frac{3}{2} \log 2$ is chosen so that $\sigma_{, u}\left(u_{i}, v_{i}\right)$ is small when $\sigma\left(u_{i}, v_{f}\right) \approx-\frac{1}{2} \log 2$. With this condition, $\sigma\left(u, v_{f}\right)$ is damped towards the desired value $\log \left(2 r_{, v}\left(u, v_{f}\right)\right)-$ $\frac{3}{2} \log 2$ along the evolution in $u$. Additionally, in order to satisfy the constraint equation (A4), we must introduce an additional ODE for the initial condition $r_{, u}\left(u, v_{i}\right)$ at $v=v_{i}$,

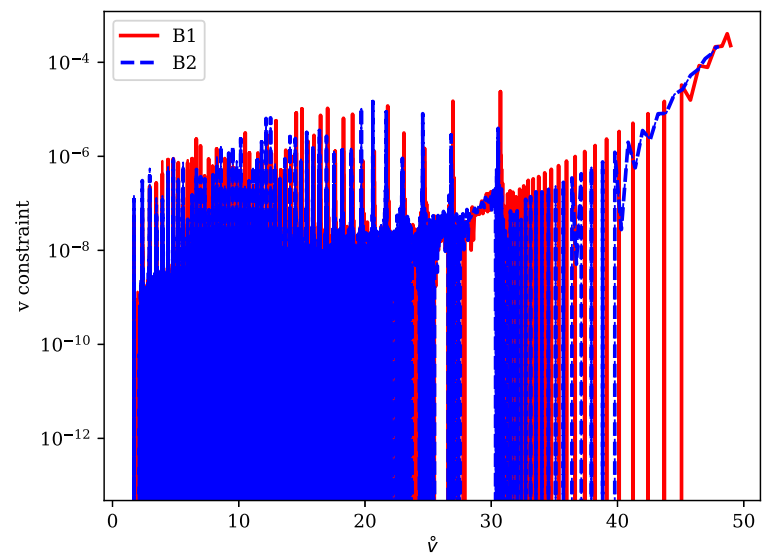

FIG. 7. Constraint violations during our evolutions of configurations B. 


$$
r_{\text {,ии }}\left(u, v_{i}\right)=2 r_{, u}\left(u, v_{i}\right) \sigma_{, u}\left(u, v_{i}\right)
$$

with $r_{, u}\left(u_{i}, v_{i}\right)=r_{u 0}$ obtained using the expression for the Misner-Sharp mass in the main text. By solving this ODE along with all the others, we get the initial conditions at $v=v_{i}$ at each value of $u$ along the integration.

\section{Code tests}

As a test of our numerical implementation we have reproduced the late-time decay of an asymptotically flat configuration with a massless scalar field. For this, it was crucial to employ the gauge conditions (A7) and (A8). We also compute the "local power" of the scalar field decay, defined as $-v \Phi_{, v} / \Phi$. These are shown in Fig. 5 and are consistent with expected results.

To further test the code, we have analyzed its convergence properties. We thus evaluate the quantity

$$
\delta_{n, m}(F) \equiv \max \left|1-F_{n} / F_{m}\right|
$$

for a given function $F_{N}$ obtained with resolution $N$ at a fixed $u$ coordinate, and where the maximum is evaluated for all values of $v$. Here, the index $m$ refers to a reference solution obtained using a large number $m$ of grid points while $n$ denotes test solutions using a coarser resolution, $n<m$.

In Fig. 6 we show the convergence properties of the Kretschmann scalar for configurations B1. The plots show exponential convergence up to $N \approx 40$.

Finally, since we use a free-evolution scheme, we have checked that the constraint equations (A4) and (A5) remain satisfied throughout our evolution. We show typical plots for the corresponding constraint violation in Fig. 7.

\section{APPENDIX B: THE KRETSCHMANN SCALAR}

To avoid round-off errors we use the expression for the Kretschmann scalar in [26] (adapted to include a scalar field mass), instead of computing it directly in terms of the metric:

$$
\begin{aligned}
K \equiv & R_{\alpha \beta \gamma \delta} R^{\alpha \beta \gamma \delta}=\frac{16}{r^{6}}\left[\left(M-\frac{3 Q^{2}}{2 r}+\frac{\Lambda}{6} r^{3}\right)+\frac{r}{2}\left(1-\frac{2 M}{r}+\frac{Q^{2}}{r^{2}}-\frac{\Lambda}{3} r^{2}\right)\left(\frac{r \Phi_{, u}}{r_{, u}}\right)\left(\frac{r \Phi_{, v}}{r_{, v}}\right)\right]^{2} \\
& +\frac{16}{r^{6}}\left(M-\frac{Q^{2}}{2 r}+\frac{\Lambda}{6} r^{3}\right)^{2}+\frac{16}{r^{6}}\left(M-\frac{Q^{2}}{r}-\frac{r^{3}}{3}\left(\Lambda+\mu^{2} \Phi^{2}\right)\right)^{2}+\frac{4}{r^{4}}\left(1-\frac{2 M}{r}+\frac{Q^{2}}{r^{2}}-\frac{\Lambda}{3} r^{2}\right)^{2}\left(\frac{r \Phi_{, u}}{r_{, u}}\right)^{2}\left(\frac{r \Phi_{, v}}{r_{, v}}\right)^{2},
\end{aligned}
$$

where $M$ is the Misner-Sharp mass.

\section{APPENDIX C: THE SCALAR FIELD ALONG THE EH}

The scalar field along the event horizon is shown in Fig. 8 (contrast with the asymptotically flat example of Fig. 5). The late-time behavior is described by ringing exponential falloff of the signal, well described by the lowest quasinormal modes of the spacetime [4].
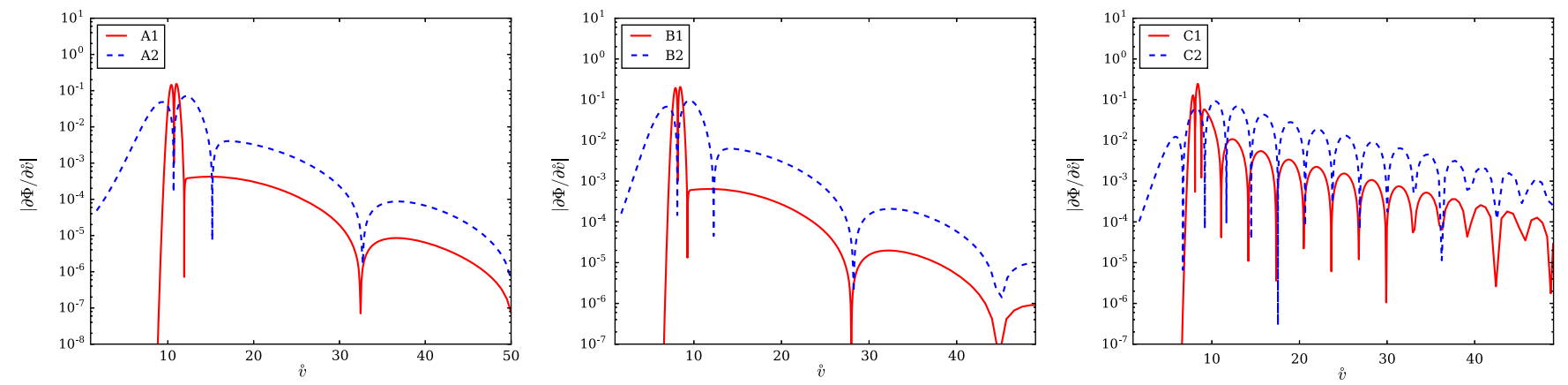

FIG. 8. Scalar field derivative $\partial \Phi / \partial \stackrel{\circ}{v}$ as a function of $\stackrel{\circ}{v}$ for configurations A (left plot), B (middle plot), and $\mathbf{C}$ (right plot) with initial profiles 1 (red solid lines) and 2 (blue dashed lines). $\partial \Phi / \partial \stackrel{\circ}{v}$ evaluated at $u=u_{\mathrm{EH}}$. 
[1] J. L. Costa, P. M. Girão, J. Natário, and J. D. Silva, Commun. Math. Phys. 361, 289 (2018).

[2] J. L. Costa and A. T. Franzen, Ann. Inst. Henri Poincaré 18, 3371 (2017).

[3] P. Hintz and A. Vasy, J. Math. Phys. (N.Y.) 58, 081509 (2017).

[4] V. Cardoso, J. L. Costa, K. Destounis, P. Hintz, and A. Jansen, Phys. Rev. Lett. 120, 031103 (2018).

[5] V. Cardoso, J. L. Costa, K. Destounis, P. Hintz, and A. Jansen, Phys. Rev. D 98, 104007 (2018).

[6] O. J. C. Dias, H. S. Reall, and J. E. Santos, J. High Energy Phys. 10 (2018) 001.

[7] Y. Mo, Y. Tian, B. Wang, H. Zhang, and Z. Zhong, Phys. Rev. D 98, 124025 (2018).

[8] O. J. C. Dias, H. S. Reall, and J.E. Santos, Classical Quantum Gravity 36, 045005 (2019).

[9] F. Mellor and I. Moss, Phys. Rev. D 41, 403 (1990).

[10] P. R. Brady, I. G. Moss, and R. C. Myers, Phys. Rev. Lett. 80, 3432 (1998).

[11] M. Dafermos, Commun. Pure Appl. Math. 58, 0445 (2005).

[12] J. Luk, J. Am. Math. Soc. 31, 1 (2018).

[13] M. Dafermos and J. Luk, arXiv:1710.01722.

[14] J. Luk and S.-J. Oh, arXiv:1702.05715.

[15] J. Luk and S.-J. Oh, arXiv:1702.05716.

[16] M. Van de Moortel, Commun. Math. Phys. 360, 103 (2018).
[17] M. Dafermos, Commun. Math. Phys. 332, 729 (2014).

[18] S. Klainerman, I. Rodnianski, and J. Szeftel, Inventiones Mathematicae 202, 91 (2015).

[19] A. Ori, Phys. Rev. Lett. 67, 789 (1991).

[20] L. M. Burko and A. Ori, Phys. Rev. D 56, 7820 (1997).

[21] J. Hansen, A. Khokhlov, and I. Novikov, Phys. Rev. D 71, 064013 (2005).

[22] P. P. Avelino, A. J. S. Hamilton, C. A. R. Herdeiro, and M. Zilhao, Phys. Rev. D 84, 024019 (2011).

[23] J. Sbierski, J. Diff. Geom. 108, 319 (2018).

[24] P. R. Brady, C. M. Chambers, W. Krivan, and P. Laguna, Phys. Rev. D 55, 7538 (1997).

[25] E. Poisson and W. Israel, Phys. Rev. Lett. 63, 1663 (1989).

[26] J. L. Costa, P. M. Girão, J. Natário, and J. D. Silva, Ann. PDE 3, 8 (2017).

[27] M. Dafermos and Y. Shlapentokh-Rothman, Classical Quantum Gravity 35, 195010 (2018).

[28] O. J. C. Dias, F. C. Eperon, H. S. Reall, and J. E. Santos, Phys. Rev. D 97, 104060 (2018).

[29] J. Bezanson, A. Edelman, S. Karpinski, and V. B. Shah, SIAM Rev. 59, 65 (2017).

[30] C. Rackauckas and Q. Nie, J. Open Res. Software 5, 15 (2017).

[31] E. Eilon and A. Ori, Phys. Rev. D 93, 024016 (2016).

[32] R. H. Price, Phys. Rev. D 5, 2419 (1972). 